\title{
New Frontiers and Innovative Methods for Hybrid Simulation
}

\section{S.J. Dyke ${ }^{1} \cdot$ O.S. Bursi ${ }^{2} \cdot$ B. Stojadinovic ${ }^{3}$}

Received: 21 August 2020 / Published online: 11 September 2020

(C) The Society for Experimental Mechanics, Inc 2020, corrected publication 2020

Resilient infrastructure requires that we strive to obtain a deeper understanding of the salient behaviors and uncertainties present when structures are exposed to extreme or hazardous loading conditions. When structural systems are too large or complex to test in the laboratory, the cyberphysical testing method known as hybrid simulation (HS), a.k.a. dynamic virtualization, provides an important tool for their examination. By coupling the physical and virtual subsystems is especially beneficial due to the realistic conditions that can be imposed on the physical subsystem.

Past applications of hybrid simulation have largely focused on addressing issues in earthquake engineering. However, in the last several years, we have seen an explosion in novel applications in which hybrid simulation methods are being exploited. Researchers are advancing the theory and practice of hybrid simulation to consider a spectrum of scenarios such as severe wind loading, coastal inundation and tsunami loading, seismic-induced fires, thermo-mechanical loading, and even bio-mechanical systems. In parallel, researchers have also been pushing the boundaries to examine approaches to revitalize hybrid simulation such as experimental design, machine learning, uncertainty quantification, nonlinear and adaptive control, decoding instabilities, and parallel computing. This timely and tremendous expansion in the scope and capabilities of this class of methods is driven by the need to investigate, at lower cost, both multi-physics and multi-hazard scenarios, while also performing experiments to establish rigorous solutions to future infrastructure challenges.

In 2017 the US National Science Foundation funded a virtual community, the Multi-hazard Engineering Collaboratory on Hybrid Simulation (MECHS) to bring together researchers in hybrid simulation from around the world for a series of

S.J. Dyke

sdyke@purdue.edu

Purdue University, West Lafayette, IN, USA

University of Trento, Trento, Italy

ETH Zurich, Zurich, Switzerland international workshops. The 2nd MECHS Workshop "New Frontiers and Innovative Methods" was held on March 13-15, 2019 in Zurich, Switzerland in partnership with researchers at ETH Zurich. Thirty-five researchers participated, including multi-hazard engineering researchers, graduate students, international partners and interdisciplinary collaborators. This special issue is meant to share the research directions discussed during this joint ETH-MECHS workshop to advance the field of hybrid simulation and to explore new and challenging applications.

The special issue begins with an article focused on the fidelity of these coupled simulation methods. Sauder examines the influence of the control system that enforces the interface conditions. Through a systematic study he considered the effects of uncertainty, noise and delays on the ability of the cyber-physical test methods to replicate the behavior of the full structural system.

Fire testing is evolving into an upcoming topic for making advances in hybrid simulation methods. Mergny et al. examined the stability of such tests as the stiffness of the structural members evolves under fire hazards and developed proportional-integral control algorithms that are sufficiently robust to compensate for those changes. Renard et al. also examined RTHS for fire hazards, and developed adaptive controller to track the displacement command. Both of these studies considered virtual RTHS environments for illustrating their methods.

Computational bottlenecks in the implementation of RTHS methods limit the range of RTHS tests, and are thus being considered by various researchers. Bas and Moustafa experimentally examined the computational load and suitability of various nonlinear integration schemes for application in RTHS. They considered a variety of integration schemes and compared them in terms of their use for real time execution while considering bracing schemes suitable for retrofits. Further, Condori et al. implemented real time parallel computing using the CyberMech platform. They developed an approach that uses sliding mode control and nonlinear Bayesian estimators, executed in parallel on a multi-core 
processor, to deal with the uncertainty and modeling errors present in the physical specimen.

The ability to quantify the uncertainty present in a particular outcome is an important question. Due to the relevance of this topic in many fields, there has been a great deal of research in this topic in recent years.Chen et al., explore the use of uncertainty quantification methods to account for variability in both ground motions and parameters. They compare several methods commonly used for uncertainty quantification to quantify the uncertainty in test outputs. Ligeikis and Christenson consider innovative ways to develop metamodels which can replace computationally intensive numerical components for use in RTHS. In geographically-distributed realtime hybrid simulation a significant source of uncertainty is the network delay. Sadraddin et al., seek to advance distributed methods for RTHS, and here they evaluate the performance of their delay compensation methods to deal with network delays in both virtual and experimental environments. In order to shed light on the seismic response of complex industrial plants, Sayginer et al. presented a methodology capable of assessing the vulnerability characteristics of a realistic tank- piping system based on hybrid simulation, synthetic ground motions and acoustic emission analysis.

The examination of mechanical systems can also benefit from the use of hybrid simulation testing. Ruffini et al. applied RTHS methods to study aerodynamic gust loading in strut-braced wings and the potential benefits of devices that are designed to alleviate vibration. Here electrodynamic shakers were used as an alternative to the commonly used hydraulic devices in previous experiments, and the goal was to explore the design space with a low-cost testing approach. Bartl et al. proposed the use of a power flow approach to design a stabilizing adaptive controller for RTHS experiments. The validate the approach using a physical component that includes a Duffing spring as the nonlinearity, and the results demonstrate that the adaptive controller is able to prevent damaging behavior.

Publisher's Note Springer Nature remains neutral with regard to jurisdictional claims in published maps and institutional affiliations. 